\title{
Estimación de Parámetros Cinéticos de la Degradación Aeróbica de Efluentes Lácteos usando AQUASIM v 2.1b
}

\author{
Miguel A. Rosa, José M. Peralta y Diego M. Bosco \\ Universidad Tecnológica Nacional - Facultad Regional Villa María, Grupo de Investigación en \\ Simulación para Ingeniería Química (GISIQ), Avda. Universidad 450 - (X5900HLR) Villa María, \\ Córdoba-Argentina (e-mail: rosa_m@frvm.utn.edu.ar)
}

Recibido Oct. 26, 2009; Aceptado Ene. 20, 2010; Versión Final recibida Feb. 24, 2010

\begin{abstract}
Resumen
Se estimaron parámetros cinéticos del mecanismo de biodegradación aeróbica de efluentes lácteos, empleando técnicas de simulación, estimación de parámetros y análisis sensitivo, disponibles en el software Aquasim v 2.1b. Se utilizó un reactor tanque agitado discontinuo de 4 litros de capacidad con inyección de aire permanente. El consumo de sustrato se representó considerando cinética de primer orden en un caso y del tipo de Monod en el otro. Se obtuvo un elevado valor de la constante de Monod, $\mathrm{K}_{\mathrm{S}}$, lo cual limitó el análisis a la primera alternativa. Los valores hallados de los parámetros de respiración endógena $\mathrm{k}_{d} \mathrm{y}$ síntesis celular $\mathrm{Y}$, fueron similares en ambos casos, mientras que el análisis sensitivo determinó parámetros de Monod no identificables. Se concluye que para este caso resulta válida una cinética de primer orden para representar el consumo de sustrato y se destaca la importancia de esta herramienta para efectuar el análisis.
\end{abstract}

Palabras claves: efluentes lácteos, biodegradación, parámetros cinéticos, análisis de sensitividad

\section{Estimation of Kinetic Parameters of the Aerobic Degradation of Dairy Wastewater using AQUASIM v 2.1b}

\begin{abstract}
The kinetic constants of an aerobic biodegradation mechanism of dairy wastewaters were estimated using techniques of simulation, estimation of parameters and sensitivity analysis, available in the software Aquasim v 2.1b. A discontinuous, agitated reactor, of 4 liters capacity with a permanent air injection was used. Two kinetic models, Monod and first order were considered to represent substrate consumption as a function of time. Due to a high value of Monod's constant, $\mathrm{K}_{\mathrm{s}}$, the data analysis was restricted to the first kinetic proposed. The values obtained for the parameters of endogenous breathing $k_{d}$ and cellular synthesis $\mathrm{Y}$, were similar, whereas the sensitivity analysis revealed nonidentifying Monod parameters. The importance of this software to carry out the analysis shown above and the validation of the first order kinetic for representing the substrate consumption are two of the most important conclusions of this work.
\end{abstract}

Keywords: dairy wastewater, substrate degradation, kinetic parameters, sensitivity analysis 


\section{INTRODUCCIÓN}

La complejidad de los procesos de tratamiento de aguas residuales en general se ha incrementado con el transcurso del tiempo, y en consecuencia los modelos matemáticos que los representan han tenido un mayor nivel de desarrollo para controlar y predecir mejor el comportamiento de las plantas depuradoras. El modelado del consumo de substrato y crecimiento celular en estos sistemas es sumamente complejo en general, y más aún en el caso particular de efluentes de la industria láctea, tal como lo evidencian numerosas publicaciones (Carta-Escobar et. al, 2005; Sage et. al, 2006). Adsorción, catálisis enzimática, y procesos difusionales son los principales mecanismos funcionales que controlan el consumo de un substrato específico, los que además dependen de variables biológicas y fisicoquímicas del sistema. En general, ajustando los parámetros del modelo se puede lograr una buena correlación de los datos experimentales, no obstante lo cual un set de parámetros diferentes también puede correlacionar tales valores de manera adecuada (Jeppsson, 1996).

El diseño de los sistemas aerobios de tratamiento de aguas residuales se realiza en base a parámetros biocinéticos que deben determinarse experimentalmente utilizando el efluente a tratar, no siendo recomendable emplear simplemente los datos reportados en la bibliografía (Martínez y Rodríguez, 2005). Se ha comunicado un importante número de trabajos en los que se describe la metodología empleada para la determinación de dichos parámetros, siendo las más utilizadas las técnicas clásicas reportadas por Eckenfelder y Wesley (1980). También existen diferentes métodos respirométricos señalados en la literatura (Carrión et. al., 2003; Gearney et. al., 2001; Kong et. al., 1994), los cuales se basan en la obtención de lecturas de oxígeno disuelto, que son luego procesadas para la obtención de información de interés. Entre las diversas técnicas respirométricas reportadas, se encuentra la de pulsos (Gearney et. al., 2001), la que además de efectuar la estimación de parámetros cinéticos, permite observar la actividad biológica de microorganismos heterótrofos y autótrofos (Brouwer et. al., 1998, Carrión et. al., 2005). Por otro lado, el creciente desarrollo de la simulación de procesos, indujo al diseño de programas de computación capaces de efectuar la simulación propiamente dicha y de juzgar la identificabilidad de los parámetros del modelo, estimando sus valores a partir de los datos medidos y la incertidumbre de los resultados calculados (Reichert, 1998).

En actividades anteriores dentro de la misma línea temática, los autores han trabajado en la comparación y selección de estructuras de modelo para la biodegradación aeróbica de efluentes industriales en general y de la industria láctea en particular (Rosa et. al., 2008a, 2008b). En el presente trabajo se ha realizado la estimación de algunos de los parámetros cinéticos correspondientes a la biodegradación aeróbica de sustrato y crecimiento de biomasa de los efluentes lácteos de una importante industria regional, empleando técnicas de simulación, estimación de parámetros y análisis de sensibilidad disponibles en un software específico de simulación e identificación de sistemas acuáticos, Aquasim v 2.1b.

\section{MATERIALES Y MÉTODOS}

La actividad experimental se llevó a cabo en un reactor tanque agitado discontinuo de 4 litros de capacidad con inyección de aire permanente para mantener la condición de mezcla completa durante toda la experiencia. Las variables evaluadas en función del tiempo fueron: demanda química de oxígeno (DQO), sólidos totales (ST) y sólidos suspendidos volátiles en el licor mezclado (MLVSS). Los resultados obtenidos se presentan en la Tabla 1.

Tabla 1: Valores experimentales vs. tiempo

\begin{tabular}{|l|c|c|c|c|c|c|c|c|c|}
\hline Tiempo (h.) & 0.0 & 6.5 & 22.0 & 28.5 & 49.0 & 120.5 & 178.0 & 230.0 & 298.0 \\
\hline DQO (mg/l) & 4730.9 & 4300.8 & 2580.5 & 2422.5 & 1009.9 & 427.4 & 527.2 & 235.3 & - \\
\hline ST $(\mathrm{mg} / \mathrm{l})$ & 1970 & - & 2705 & 3380 & 3005 & 2900 & - & 2320 & 2650 \\
\hline MLVSS (mg/l) & 1445 & - & 2535 & 2835 & 2785 & 2690 & - & 2230 & 1940 \\
\hline
\end{tabular}




\section{Características generales del software Aquasim v 2.1b}

Aquasim fue diseñado para la identificación y simulación de sistemas acuáticos en laboratorios, plantas industriales y ámbitos naturales. El programa permite configurar cuatro etapas: simulación, análisis de confiabilidad, estimación de parámetros y análisis de incertidumbre.

En la primera etapa el usuario lleva a cabo la simulación del modelo implementado lo cual le permite establecer, por comparación de los resultados calculados con los valores medidos, si las hipótesis formuladas en el modelo son compatibles. La observación de desviaciones sistemáticas entre los resultados de la simulación y los valores determinados experimentalmente implica de manera indirecta que deban considerarse otros procesos adicionales o bien que es necesario reformular el modelo propuesto. Aquasim permite al usuario cambiar fácilmente la estructura del modelo y valores de los parámetros. La segunda etapa consiste en conformar el análisis sensitivo con respecto al set de variables seleccionadas. Esta etapa brinda al usuario la posibilidad de calcular funciones sensitivas lineales de variables arbitrarias con respecto a cada uno de los parámetros incluidos en el análisis. Tales funciones aportan a la evaluación de la identificabilidad de los parámetros del modelo. Además, las derivadas calculadas en este análisis, permiten estimar la incertidumbre en cualquier variable de acuerdo a la fórmula de propagación de error lineal. El cálculo de la contribución de cada parámetro a la incertidumbre total facilita la detección de mayores fuentes de desviaciones. La tercera etapa de suma importancia es la realización de la estimación de parámetros automáticamente para una estructura de modelo dado utilizando datos medidos. Esto no solamente es importante para obtener estimaciones neutras de los parámetros, sino que constituye el principal requisito para comparar de manera eficiente modelos diferentes. Los parámetros son estimados por el software a través de la minimización de la suma de los cuadrados de las desviaciones medias entre los valores medidos y los resultados arrojados por el modelo, la cual se determina por:

$\chi^{2}(p)=\sum_{i=1}^{n}\left(\frac{y_{\text {meas }, i}-y_{i}(p)}{\sigma_{\text {meas }, i}}\right)^{2}$

Donde $y_{\text {meas, }}$ : i-ésima medida; $\sigma_{\text {meas,i: }}$ : desviación estándar de la i-ésima medida; $y_{i}(p)$ : valor calculado de la variable del modelo correspondiente a la i-ésima medida, evaluada al tiempo y localización de la misma; $p=\left(p_{1}, \ldots, p_{m}\right)$ : parámetros del modelo y $n: n^{\circ}$ de determinaciones. El software ejecuta la minimización del valor de $\chi^{2}$ sujeta a la condición: $p_{\text {mín,i }} \leq p_{i} \leq p_{m a ́ x, i}$, siendo $p_{\text {mín,i }}$ y $p_{\text {máx,i }}$ los valores mínimos y máximos de la variable constante $\left(p_{i}\right)$, respectivamente, que deben ser debidamente especificados. La determinación cuantitativa de la desviación entre los resultados calculados y los medidos, la cual es minimizada por el algoritmo de estimación de parámetros, es útil para evaluar la suficiencia del modelo estadísticamente.

\section{Formulación del modelo}

En el programa, un modelo consiste de un sistema de ecuaciones diferenciales ordinarias y/o a derivadas parciales y ecuaciones algebraicas, las que describen el comportamiento de un set de variables de estado de un sistema acuático. Las ecuaciones diferenciales para flujo de agua y transporte de substancias pueden seleccionarse con la opción de compartimientos, y las condiciones iniciales de las mismas, pueden especificarse libremente por el usuario. La definición de los procesos, compartimientos y enlaces, se hace con la ayuda de variables que representan objetos con un valor numérico posible en el contexto sensitivo. Es evidente que las variables, de las cuales se distinguen seis tipos, constituyen el subsistema básico para la formulación de los restantes. Los procesos (dinámico o de equilibrio) deben definirse antes de que los mismos sean activados en compartimientos. Por último, los enlaces se utilizan para conectar los compartimientos configurados. Los cuatro subsistemas señalados, una vez definidos constituyen el modelo utilizado para la simulación y análisis de datos (Reichert, 1998). 
Para el caso planteado se consideraron dos variables de estado, el consumo de materia orgánica y el crecimiento de biomasa. Se configuró un proceso dinámico con dos situaciones alternativas: cinética de primer orden en un caso y del tipo de Monod en el otro. El compartimiento seleccionado fue el reactor de mezcla completa. En primer lugar se evaluó la condición de cinética de primer orden para el consumo de sustrato, hallándose que la mejor correlación de los resultados experimentales se obtuvo para un valor del parámetro de síntesis celular (Y), de 0.866. Luego, utilizando este valor, el mismo modelo se formuló planteando una cinética del tipo de Monod para representar la degradación de materia orgánica. Los parámetros cinéticos determinados mediante simulación y estimación de parámetros fueron: la constante de velocidad de reacción para el modelo de $1^{\circ}$ orden $(\mathrm{k})$, la velocidad específica máxima $\left(u_{\text {máx }}\right)$ y constante de saturación $\left(\mathrm{K}_{\mathrm{S}}\right)$ para el modelo de Monod, y los parámetros de producción de biomasa (respiración endógena: $\mathrm{k}_{\mathrm{d}}$, y síntesis celular: $\mathrm{Y}$ ).

Para el análisis sensitivo se utilizó la función sensibilidad absoluta-relativa (SensAR), definida por:

SensAR $(D Q O, p)=p \frac{\partial(D Q O)}{\partial p}$,

Donde $\mathrm{p}$ representa el parámetro.

\section{RESULTADOS Y DISCUSIÓN}

En Tabla 2 y Fig. 1 y 2, se presentan los resultados obtenidos por simulación y estimación de parámetros, respectivamente, para ambos modelos.

Tabla 2: Valores estimados de los parámetros biocinéticos

\begin{tabular}{|c|c|c|c|c|c|}
\hline \multicolumn{5}{|c|}{ PROCESO } & \multirow{4}{*}{$\begin{array}{c}\text { FACTOR DE } \\
\text { CORRELACIÓN } \\
\left(\chi^{2}\right)\end{array}$} \\
\hline DEGRADAC & DN DE SUST & ATO & \multirow{2}{*}{\multicolumn{2}{|c|}{ CRECIMIENTO DE BIOMASA }} & \\
\hline Cinética $1{ }^{\circ}$ orden & Cinética tip & Monod & & & \\
\hline k (l/mg.h) & $u_{\text {máx }}(\mathrm{l} / \mathrm{mg} \cdot \mathrm{h})$ & $\mathrm{K}_{\mathrm{S}}(\mathrm{mg} / \mathrm{l})$ & $k_{d}(1 / h)$ & $\mathrm{Y}$ & \\
\hline 0.0164 & - & - & 10.446 & 0.866 & 47.2 \\
\hline- & 249.4 & 11867.3 & 10.260 & 0.866 & 47.9 \\
\hline
\end{tabular}

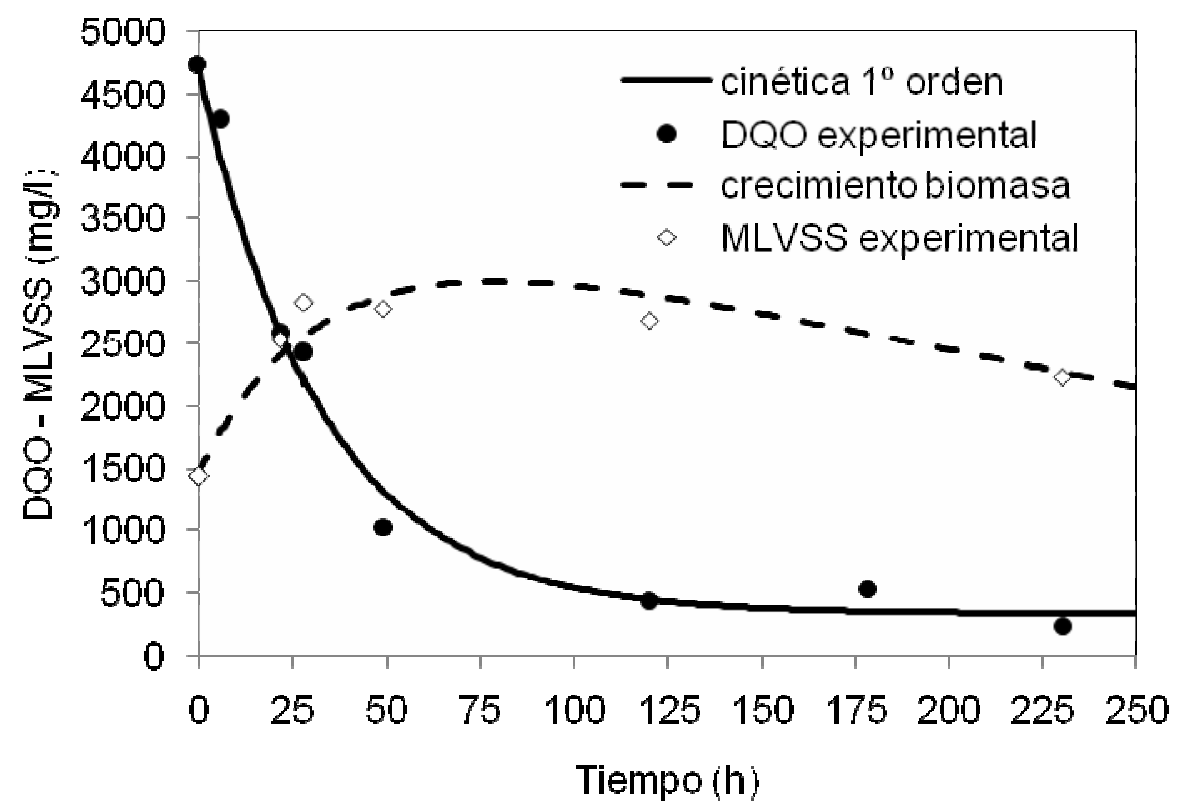

Fig. 1: Resultados simulación cinética $1^{\circ}$ orden vs. valores experimentales 


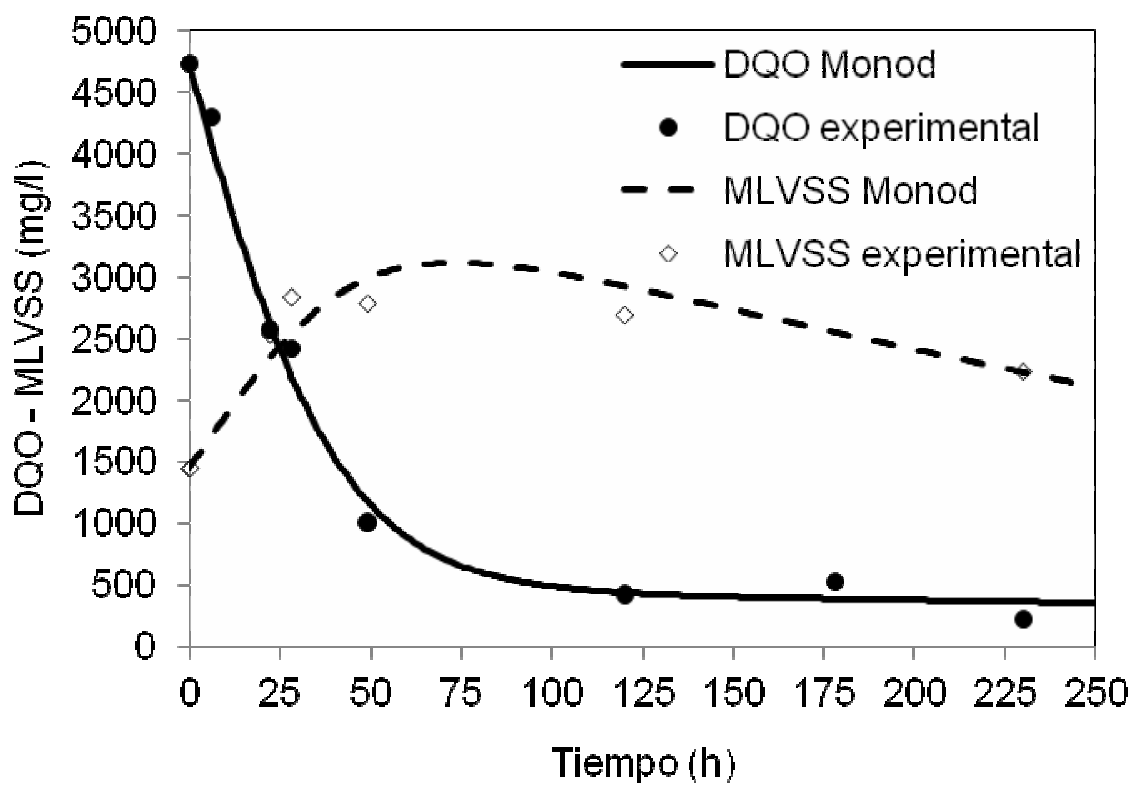

Fig. 2: Resultados simulación cinética de Monod vs. valores experimentales

El factor de correlación obtenido, prácticamente igual para ambos modelos, se explica considerando el elevado valor de la constante de saturación de Monod $\left(\mathrm{K}_{\mathrm{S}}\right)$, lo cual permite inferir que en la expresión de Monod: $u=u_{\text {máx }} \mathrm{S} /\left(\mathrm{K}_{\mathrm{S}}+\mathrm{S}\right)$, la concentración de sustrato puede despreciarse en el denominador, transformando la misma en una cinética de primer orden. Por esta razón el análisis se limita a evaluar solamente la primera alternativa. Esto se hace aún más evidente al observar que en ambos casos el valor obtenido de la constante $\mathrm{k}_{\mathrm{d}}$, es prácticamente el mismo. Además de lo anterior, los resultados del análisis sensitivo indican que los parámetros del modelo de Monod no resultan identificables puesto que, según lo observado en la Fig. 3, las funciones sensitivas de $K_{s}$ y $\mu_{\text {máx }}$ tienen una forma similar lo cual indica una relación de dependencia lineal entre estos parámetros, es decir que variaciones de la concentración calculada para un tiempo dado, producidas por un cambio en el valor de $\mathrm{K}_{\mathrm{s}}$, pueden aproximadamente ser compensadas por una modificación apropiada del valor de $\mu_{\text {máx }}$ o viceversa (Reichert, 1998). Esto permite asegurar que no existe un único par de valores $\left(K_{s}, \mu_{\text {máx }}\right)$ que satisfaga la expresión de Monod lo cual no garantiza la confiabilidad del modelo puesto que la interpretación física del mismo para cada par de valores de los parámetros sería diferente. Esta situación no se presenta en el caso de cinética de $1^{\circ}$ orden puesto que el análisis se limita a un único parámetro, la constante de velocidad de reacción (k), el cual resulta identificable considerando los elevados valores de la función SensAR, básicamente en la primera etapa del proceso de biodegradación.

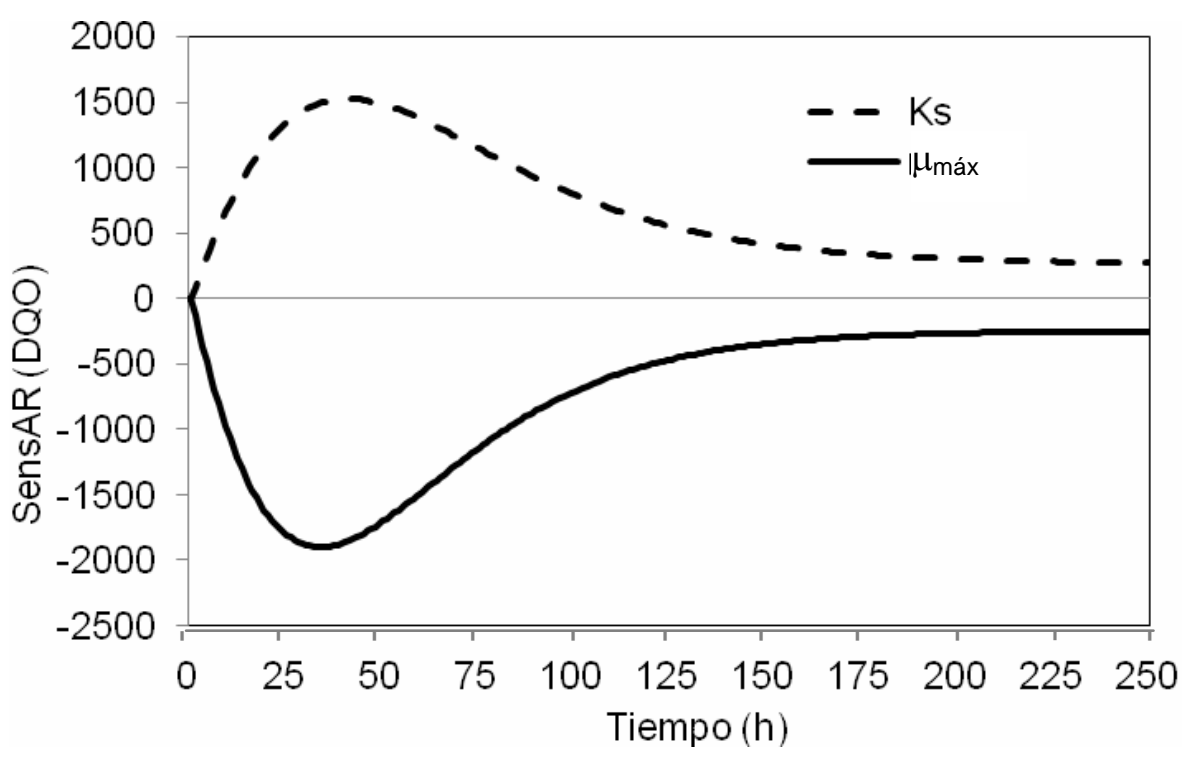

Fig. 3: Análisis de sensibilidad modelo de Monod 


\section{CONCLUSIONES}

En virtud de lo expresado en la sección anterior se pueden establecer las siguientes conclusiones:

Para el caso particular evaluado los resultados experimentales correspondientes al consumo de sustrato y crecimiento celular pueden ser representados de manera adecuada mediante el modelo cinético de primer orden, puesto que utilizando una cinética de mayor complejidad como la del tipo de Monod no se consigue una mejora en el factor de correlación y además sus parámetros no resultan identificables lo cual le resta confiabilidad a este modelo.

En términos generales se resalta la importancia de la herramienta informática y particularmente del software empleado en este caso para llevar a cabo este tipo de análisis y efectuar la determinación de parámetros biocinéticos de la degradación aeróbica de efluentes industriales.

\section{REFERENCIAS}

Brouwer H., A. Klanpwijk y K. J. Keesman; Identification of Activated Sludge and Wastewater Characteristics using Respirometric Batch-Experiments; Water Research: 32(4), 1240-1254, (1998).

Carrión M., A. Assaf y F. Thalasso; Respiration Rate Measurement in Submerged Fixed Bed Reactor; Water Science Technology.: 47(5), 201- 204, (2003).

Carrión M., J. Alba y F. Thalasso; Effect of Hydrodynamic Conditions on Biofilms Oxygen Consumption Rate in a Fixed-Bed Nitrifying Reactor; Water Science Technology: 52(7), 91-95 (2005).

Carta-Escobar F., y otros cuatro autores; Aerobic Purification of Dairy Wastewater in Continuous Regime: Part II: Kinetic Study of the Organic Matter Removal in Two Reactor Configurations; Biochemical Eng. Journal: 22(2), 117-124 (2005).

Eckenfelder W. y J. Wesley; Principles of Water Quality Magnagment; 131-142, Pumberton, Boston, MA, EEUU (1980).

Gearney A. K., B. Petersen, J. P. Ottoy y P. Vanrolleghem; Activated Sludge Monitoring with Combined Respirometrictritimetric Measurements; Water Research: 35(5), 1280-1294 (2001).

Jeppsson U.; Modelling Aspects of Wastewater Treatment Processes; Ph.D. dissertation, IEA, Lund Institute of Technology, (ISBN 91-88934-00-4), Lund, Sweden (1996).

Kong Z., P. Vanrolleghem, P. Willens y W. Verstraete; Simultaneous Determination of Inhibition Kinetics of Carbón Oxidation and Nitrification with a Respirometer; Wat. Res.: 30 (4), 825-836 (1996).

Martínez Delgadillo S. A. y M. G. Rodríguez Rosales; Tratamiento de Aguas Residuales con Matlab; $1^{\circ}$ Edición, 124-153, Ed. Reverté, Universidad Autónoma Metropolitana, México (2005).

Reichert P.; Aquasim 2.0. User Manual. Computer Program for the Identification and Simulation of Aquatic Systems; 2-10, Swiss Federal Institute for Environmental Science and Technology (EAWAG), Dübendorf, Suiza (1998).

Rosa M. A., J. Peralta, D. Gilli y D. Bosco; Cinética de la Biodegradación de un Efluente Lácteo. Aplicación de Técnicas de Análisis de Datos para Comparación de Modelos; Información Tecnológica, Centro de Información Tecnológica: 19(4), 11-18 (2008).

Rosa M. A., D. Gilli y D. Bosco; Aplicación de Técnicas de Análisis de Datos para Comparación de Modelos Cinéticos de la Biodegradación de Efluentes Industriales; Ing. Alimentaria: 75, 58-64 (2008).

Sage M., G. Daufin y G. Gésan-Guiziou; Denitrification Potential and Rates of Complex Carbon Source from Dairy Effluents in Activated Sludge System; Water Research: 40(14), 2747-2755 (2006). 